\title{
Gamma-Tocotrienol Modulated Gene Expression in Senescent Human Diploid Fibroblasts as Revealed by Microarray Analysis
}

\author{
Suzana Makpol, ${ }^{1}$ Azalina Zainuddin, ${ }^{1}$ Kien Hui Chua, ${ }^{2}$ \\ Yasmin Anum Mohd Yusof, ${ }^{1}$ and Wan Zurinah Wan Ngah $^{1}$ \\ ${ }^{1}$ Department of Biochemistry, Faculty of Medicine, Universiti Kebangsaan Malaysia, Jalan Raja Muda Abdul Aziz, \\ 50300 Kuala Lumpur, Malaysia \\ ${ }^{2}$ Department of Physiology, Faculty of Medicine, Universiti Kebangsaan Malaysia, Jalan Raja Muda Abdul Aziz, \\ 50300 Kuala Lumpur, Malaysia
}

Correspondence should be addressed to Suzana Makpol; suzanamakpol@yahoo.com

Received 14 December 2012; Revised 13 February 2013; Accepted 13 February 2013

Academic Editor: Narasimham L. Parinandi

Copyright (c) 2013 Suzana Makpol et al. This is an open access article distributed under the Creative Commons Attribution License, which permits unrestricted use, distribution, and reproduction in any medium, provided the original work is properly cited.

\begin{abstract}
The effect of $\gamma$-tocotrienol, a vitamin E isomer, in modulating gene expression in cellular aging of human diploid fibroblasts was studied. Senescent cells at passage 30 were incubated with $70 \mu \mathrm{M}$ of $\gamma$-tocotrienol for $24 \mathrm{~h}$. Gene expression patterns were evaluated using Sentrix HumanRef-8 Expression BeadChip from Illumina, analysed using GeneSpring GX10 software, and validated using quantitative RT-PCR. A total of 100 genes were differentially expressed $(P<0.001)$ by at least 1.5 fold in response to $\gamma$-tocotrienol treatment. Amongst the genes were IRAK3, SelS, HSPA5, HERPUD1, DNAJB9, SEPR1, C18orf55, ARF4, RINT1, NXT1, CADPS2, COG6, and GLRX5. Significant gene list was further analysed by Gene Set Enrichment Analysis (GSEA), and the Normalized Enrichment Score (NES) showed that biological processes such as inflammation, protein transport, apoptosis, and cell redox homeostasis were modulated in senescent fibroblasts treated with $\gamma$-tocotrienol. These findings revealed that $\gamma$-tocotrienol may prevent cellular aging of human diploid fibroblasts by modulating gene expression.
\end{abstract}

\section{Introduction}

Aging is a phenomenon associated with gradual decline in biological functions. Loss of capability to divide besides loss of cellular functions in both mitotic and postmitotic cells is the characteristic of cellular aging [1]. Cellular changes that occur in the cell of an organism have direct impact on the functions of organs, systems and eventually involve the whole organism. The genetic theory of aging is proposed based on the observation that several genes affect longevity [2]. The aging process is regulated by specific genes in many organisms including yeast, C. elegans, fruit flies, and mice. In human diploid fibroblasts, several genes including inflammatory genes, cell cycle regulatory genes, cytoskeletal genes, and metabolic genes were differentially expressed [3] during replicative senescence and modifiable by dietary components such as antioxidants [4].

Human aging can be studied in vitro, specifically by using normal human diploid fibroblasts (HDFs) which undergo a limited number of cellular divisions in culture and progressively reached a state of irreversible growth arrest, a process termed as replicative senescence [1]. Senescent fibroblast cells are resistant to mitogen-induced proliferation, expressed senescence-associated $\beta$-galactosidase (SA $\beta$-gal), exhibited enlarged and flattened morphology, and showed altered gene expression [5]. Cultured human fibroblasts cells displayed age-dependent transcriptomic differences. A variety of genes involved in cell cycle regulation, immune response and inflammation, cytoskeleton, stress response, and metabolism are known to be altered during cellular senescence [6].

About 40 micronutrients consist of vitamins, essential minerals, and other compounds are required in small amount for normal metabolism and have been reported as essential components in the diet. Deficiency of macro- and micronutrients in aging is related to global impairments of immune functions, metabolic harmony, and antioxidant defense with subsequent appearance of age-related diseases [7]. The antioxidant vitamin E, usually alpha-tocopherol, 
scavenges reactive oxygen species (ROS) thus preventing oxidative damage associated with many degenerative diseases and has been suggested to act as a signaling molecule which modulates signal transduction and gene expression [8].

Vitamin E is a lipophilic vitamin, synthesized by plants, found particularly in plant seeds and oils. There are eight naturally occurring forms of vitamin $\mathrm{E}$ which are $\alpha-, \beta-$, $\gamma^{-}$, and $\delta$-tocopherol and $\alpha$-, $\beta$-, $\gamma^{-}$, and $\delta$-tocotrienol [9]. Structurally, tocotrienols are different from tocopherols by the presence of three trans double bonds in their hydrocarbon tail [10].

The prenyl side chain of tocotrienols has been postulated to be responsible for the differential membrane distribution and metabolism of tocotrienols. $\alpha$-tocotrienol possessed higher antioxidant activity against lipid peroxidation than $\alpha$ tocopherol due to a more uniform distribution in the lipid bilayer membrane providing a more efficient interaction of the chromanol ring with lipid radicals [11]. Tocotrienol also showed novel hypocholesterolemic activity [12] in addition to more recent reports suggesting that it has neuroprotective, antioxidant, anticancer and cholesterol lowering properties that often differ from the properties of tocopherols [10]. Furthermore, tocotrienol was able to delay cellular aging by preventing oxidative damage-induced telomere shortening in aged human fibroblast cells [13]. Also tocotrienol-rich fraction (TRF) has been shown to have antiaging properties by promoting cell cycle progression in senescent human fibroblast cells [5]. Recent research has focused on other biological functions of vitamin $\mathrm{E}$ that are unrelated to its antioxidant properties which include its roles in cellular signaling, gene expression, immune response, and apoptosis [9]. Therefore, this study was aimed to determine the effect of $\gamma$-tocotrienol in modulating gene expression in cellular aging of human diploid fibroblast cells.

\section{Materials and Methods}

2.1. Primary Culture of Human Diploid Fibroblast Cells and Treatment with $\gamma$-Tocotrienol. This research has been approved by the Universiti Kebangsaan Malaysia Ethics Committee (Approval Project Code: FF-104-2007). Informed written consent was obtained from the parents of all subjects. Primary human dermal fibroblasts were derived from the foreskins (removed during circumcision) of three 9to 12 -year-old boys. The samples were aseptically collected and washed several times with $75 \%$ alcohol and phosphate buffered saline (PBS) containing $1 \%$ antibiotic-antimycotic (PAA, Austria). After removing the epidermis, the pure dermis was cut into small pieces and transferred into falcon tubes containing $0.03 \%$ collagenase type I solution (Worthington Biochemical Corporation, USA). Pure dermis was digested in an incubator shaker at $37^{\circ} \mathrm{C}$ for $6-12 \mathrm{~h}$. Then, cells were rinsed with PBS before being cultured in Dulbecco Modified Eagle Medium (DMEM) containing 10\% foetal bovine serum (FBS) (PAA, Austria) and $1 \%$ antibiotic-antimycotic at $37^{\circ} \mathrm{C}$ in $5 \%$ $\mathrm{CO}_{2}$ humidified incubator. After 5-6 days, the cultured HDFs were harvested by trypsinization and culture-expanded in new T25 culture flasks (Nunc, Denmark) with expansion degree of $1: 4$. When the subcultures reached $80 \%-90 \%$ confluency, serial passaging was done by trypsinization, and the number of population doublings (PDs) was monitored until HDFs reached senescence. For subsequent experiments, cells were used at either passage 4 (young cells, $\mathrm{PD}<12$ ) and passage 30 (senescent cells, $\mathrm{PD}>55$ ).

In the subsequent experiments, treated young HDFs were incubated with $50 \mu \mathrm{M}$ palm $\gamma$-tocotrienol (Malaysian Palm Oil Board), while senescent HDFs were incubated with $70 \mu \mathrm{M} \gamma$-tocotrienol for $24 \mathrm{~h}$. Untreated cells were cultured in Dulbecco Modified Eagle Medium (DMEM) containing 10\% foetal bovine serum (FBS) (PAA, Austria). The media for the untreated cells were changed in parallel to the treated cells. Both untreated and treated cells were harvested on the same day [14].

2.2. Determination of Senescent Biomarker SA $\beta$-Gal Activity. The senescent biomarker of in vitro cell aging for HDFs (SA $\beta$-gal activity) was determined by senescent cells staining kit (Sigma, USA) according to the manufacturer's instruction. Blue staining was visible after $4 \mathrm{~h}$ of incubation with $\beta$-galactosidase staining solution containing 5 -bromo4 -chloro-3-indolyl- $\beta$-D-galactosidase (X-gal) at $37^{\circ} \mathrm{C}$. The percentage of blue cells observed in 100 cells under a light microscope was calculated.

2.3. Total RNA Extraction. Total RNA from HDFs in different treatment groups was extracted using TRI Reagent (Molecular Research Center, Cincinnati, OH) according to the manufacturer's instruction. Polyacryl carrier (Molecular Research Center) was added in each extraction to precipitate the total RNA. Extracted total RNA pellet was then washed with $75 \%$ ethanol and dried before being dissolved in RNase and DNase-free distilled water. Total RNA was stored at $-80^{\circ} \mathrm{C}$ immediately after extraction. Concentration and purity of the extracted RNA were determined by Agilent 2100 bioanalyzer (Agilent Technologies, USA). RNA with RNA integrity number (RIN) ranging from 7 to 10 and absorbance ratio of $A_{260}$ to $A_{280}$ ranging from 1.5 to 2.1 was utilized for cDNA synthesis.

2.4. Microarray Analysis. Briefly, $250 \mathrm{ng}$ of total RNA from each sample was labeled by using TotalPrep RNA Amplification Kit (Ambion, Austin, USA) for cDNA synthesis. In vitro transcription was performed to synthesise the cRNA. Single stranded cRNA was labeled by incorporating biotin16-UTP, and cRNA was generated by incubation in the hybridization oven for $14 \mathrm{~h}$ at $37^{\circ} \mathrm{C}$. After $14 \mathrm{~h}$ incubation, $750 \mathrm{ng}$ of biotin-labeled cRNA was hybridized $\left(18 \mathrm{~h}\right.$ at $\left.58^{\circ} \mathrm{C}\right)$ to Illumina's Sentrix HumanRef-8_v3 Expression BeadChips (Illumina, San Diego, USA). The hybridized biotinylated cRNA was detected with streptavidin-Cy3 (Amersham Biosciences, USA) and quantitated using Illumina's BeadStation S455 scanner (Illumina, San Diego, USA). Data was analysed using GeneSpring GX 10 software (Agilent Technologies, USA), and two-way analysis of variance (2-way ANOVA) was applied with the false discovery rate (FDR) for the selection of differentially expressed genes at significance level of $P<$ 
TABLE 1: Primers list for qRT-PCR.

\begin{tabular}{llll}
\hline Gene & Accession number & Primer sequences $\left(5^{\prime}-3^{\prime}\right)$ & PCR product size (bp) \\
\hline HSPA5 & NM_005347 & F: ggt gaa aga ccc ctg aca aa & 199 \\
& & R: gtc agg cga ttc tgg tca tt & 193 \\
HERPUD1 & F: gag cct gct ggt tct aat cg & R: gaa agc tga agc cac cca ta \\
ARF4 & NM_01010990 & F: ggg atg ttg gtg gtc aag at & 168 \\
\hline
\end{tabular}

0.001 for baseline (data not shown) and $\gamma$-tocotrienol-treated HDFs. Significant gene list was further filtered for differences of more than 1.5-fold between $\gamma$-tocotrienol-treated HDFs and untreated control senescent HDFs. Significant genes with expression greater than 1.5-fold were selected for Gene Set Enrichment Analysis (GSEA) by using pathway studio software (Ariadne, USA) with $P<0.05$.

2.5. Validation of Microarray Data Using Quantitative RealTime RT-PCR. Genes for validation were chosen from pathway analysis. Quantitative real-time RT-PCR reaction was carried out to evaluate the expression of ARF4, HSPA5, and HERPUD1 genes using $1 \mu \mathrm{L}$ total RNA as template, $1 \mu \mathrm{L}$ of forward and reverse primers for genes of interest, and iScript One-Step RT-PCR reagent with SYBR Green (Bio-Rad, USA). Primer sequences for ARF4, HSPA5, and HERPUD1 are shown in Table 1 . All reactions were run in duplicate with reaction profile as follows: CDNA synthesis for $30 \mathrm{~min}$ at $50^{\circ} \mathrm{C}$; predenaturation for $2 \mathrm{~min}$ at $94^{\circ} \mathrm{C}$; PCR amplification for 38 cycles with $10 \mathrm{sec}$ at $94^{\circ} \mathrm{C}$ and $30 \mathrm{sec}$ at $61^{\circ} \mathrm{C}$ using Bio-Rad iCycler (Bio-Rad, USA).

\section{Results}

3.1. Quality Control Assessment of the Samples. Principal Component Analysis (PCA) was used to check the quality of the microarray data. This allows viewing of separation between groups of replicates. Untreated control senescent and $\gamma$-tocotrienol-treated senescent HDFs were well separated and clustered into two distinct groups. Similar separation, however, was not observed for untreated control young and $\gamma$-tocotrienol-treated young HDFs (Figure 1).

3.2. Hierarchical Clustering of Significantly Expressed Genes. Cluster analysis was performed to organize genes into cluster based on their similarities of expression. Horizontal line represents a single gene, and each column represents a single sample. Red color indicated the upregulated genes, whereas green color indicated the downregulated genes. Statistical analysis of two-way analysis of variance (2-way ANOVA) revealed that a total of 253 genes were significantly regulated in senescent $\mathrm{HDF}$ as compared to young cells (Figure 2(a)). One hundred genes were significantly regulated in tocotrienol-treated senescent HDFs compared to untreated control senescent HDFs (Figure 2(b)). Clustering analysis was able to distinguish gene expression between young and senescent HDFs as well as between untreated

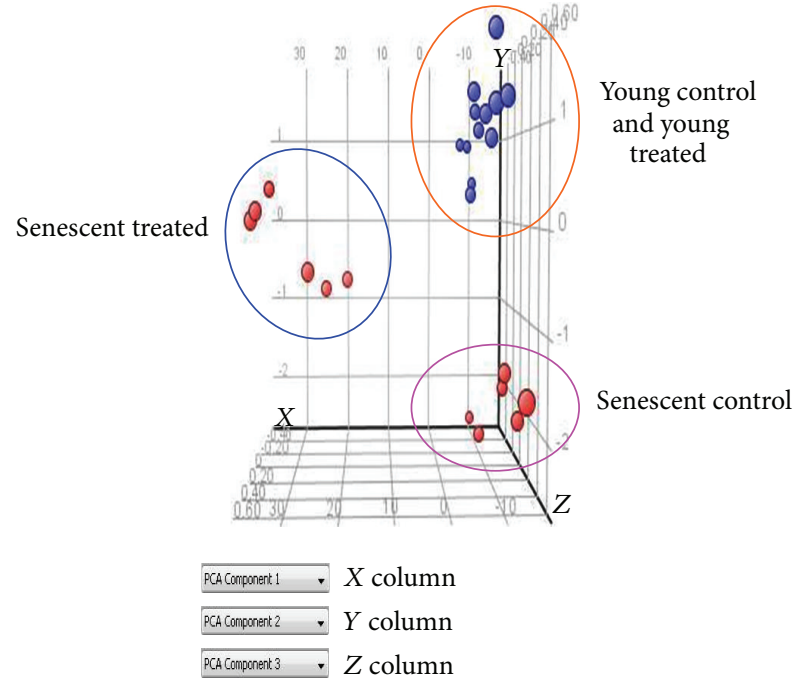

FIGURE 1: Three-dimensional Principal Component Analysis (PCA) plot was derived from biological replicates of HDFs $(n=6)$. The three dimensional PCA plot showed clustering of the different treatment groups: untreated control senescent HDFs, $\gamma$-tocotrienoltreated senescent HDFs, and untreated control young HDFs and $\gamma$ tocotrienol-treated young HDFs.

control senescent and tocotrienol-treated senescent HDFs as shown by Hierarchical clustering.

3.3. Analysis of Differentially Expressed Genes in $\gamma$-TocotrienolTreated HDFs. A total of 100 genes were differentially expressed in $\gamma$-tocotrienol-treated senescent HDFs as compared to untreated control senescent HDFs (Table 2). Gene Set Enrichment Analysis (GSEA) revealed the selected significant biological processes involved in response to $\gamma$-tocotrienol treatment in senescent HDFs compared to untreated control senescent HDFs (Table 3). Positive value of Normalized Enrichment Score (NES) indicated the upregulated process. The biological processes that were modulated by $\gamma$-tocotrienol treatment included the negative regulation of tumor necrosis factor production (inflammation), negative regulation of interleukin-6 production (inflammation), negative regulation of caspases activity (apoptosis), response to stress, transport protein, and cell redox homeostasis. Pathway analysis was carried out for selected genes (ARF4, HSPA5, and HERPUD1) (Figure 3), and validation on the microarray data was carried out by real time RT-PCR. Results showed an up-regulation of the selected genes in $\gamma$-tocotrienol-treated 
TABLE 2: Differentially expressed genes list in $\gamma$-tocotrienol-treated senescent HDFs.

\begin{tabular}{|c|c|c|c|}
\hline Gene Ontology/accession number & Gene symbol & Fold change & Regulation \\
\hline \multicolumn{4}{|l|}{ Protein transport } \\
\hline NM_020751.1 & COG6 & 2.9372108 & Upregulated \\
\hline NM_022374.1 & ARL6IP2 & 2.4375103 & Upregulated \\
\hline NM_017954.8 & CADPS2 & 2.4749646 & Upregulated \\
\hline NM_004794.2 & RAB33A & 4.2599683 & Upregulated \\
\hline NM_001660.2 & ARF4 & 3.6646543 & Upregulated \\
\hline NM_021930.3 & RINT-1 & 1.6876434 & Upregulated \\
\hline NM_001031677.2 & RAB24 & 2.3763354 & Upregulated \\
\hline NM_013248.2 & NXT1 & 3.5031867 & Upregulated \\
\hline NM_014177.1 & C18orf55 & 1.5897815 & Upregulated \\
\hline NM_017986.2 & GPR172B & 2.2754004 & Upregulated \\
\hline \multicolumn{4}{|l|}{ Ion transport } \\
\hline NM_000725.2 & CACNB3 & 2.0575228 & Downregulated \\
\hline NM_002245.2 & KCNK1 & 4.019881 & Upregulated \\
\hline NM_022055.1 & KCNK12 & 2.6714919 & Upregulated \\
\hline NM_012463.2 & ATP6V0A2 & 2.7435005 & Upregulated \\
\hline NM_005175.2 & ATP5G1 & 2.527163 & Upregulated \\
\hline NM_000785.3 & CYP27B1 & 7.801915 & Upregulated \\
\hline \multicolumn{4}{|c|}{ Negative regulation of interleukin- 6 production } \\
\hline NM_203472.1 & SELS & 2.317949 & Upregulated \\
\hline NM_007199.1 & IRAK3 & 2.630288 & Upregulated \\
\hline \multicolumn{4}{|l|}{ Immune response } \\
\hline NM_002162.2 & ICAM3 & 3.910169 & Upregulated \\
\hline \multicolumn{4}{|l|}{ Cytokine mediated signal transduction } \\
\hline NM_203453.2 & PPAPDC2 & 1.8218696 & Downregulated \\
\hline \multicolumn{4}{|l|}{ Apoptosis } \\
\hline NM_018530.1 & GSDML & 2.4119947 & Upregulated \\
\hline NM_018130.2 & SHQ1 & 1.8244729 & Upregulated \\
\hline NM_018456.4 & EAF2 & 7.062471 & Upregulated \\
\hline NM_018145.1 & FAM82C & 1.6341798 & Upregulated \\
\hline NM_024310.2 & PLEKHF1 & 2.9315765 & Upregulated \\
\hline NM_001012398.1 & AKTIP & 2.6247795 & Upregulated \\
\hline \multicolumn{4}{|l|}{ Response to stress } \\
\hline NM_005347.2 & HSPA5 & 5.951358 & Upregulated \\
\hline NM_021237.3 & SELK & 3.3043954 & Upregulated \\
\hline NM_014445.2 & SERP1 & 3.263752 & Upregulated \\
\hline \multicolumn{4}{|l|}{ Cell redox homeostasis } \\
\hline NM_016417.2 & GLRX5 & 1.7182248 & Upregulated \\
\hline \multicolumn{4}{|l|}{ Cell cycle } \\
\hline NM_006545.4 & TUSC4 & 5.8936715 & Upregulated \\
\hline NM_016948.1 & PARD6A & 2.2347567 & Upregulated \\
\hline \multicolumn{4}{|l|}{ Regulation of transcription } \\
\hline NM_005444.1 & RQCD1 & 2.0665886 & Upregulated \\
\hline NM_004634.2 & BRPF1 & 1.8511329 & Upregulated \\
\hline NM_003408.1 & ZFP37 & 2.5321555 & Upregulated \\
\hline NM_015394.4 & ZNF10 & 2.5174437 & Upregulated \\
\hline
\end{tabular}


TABLE 2: Continued.

\begin{tabular}{|c|c|c|c|}
\hline Gene Ontology/accession number & Gene symbol & Fold change & Regulation \\
\hline NM_001080485.1 & ZNF275 & 1.8915362 & Upregulated \\
\hline NM_005088.2 & CXYorf3 & 2.2247052 & Upregulated \\
\hline NM_032758.3 & PHF5A & 2.0002973 & Upregulated \\
\hline NM_022366.1 & TFB2M & 2.2978535 & Upregulated \\
\hline NM_145805.1 & ISL2 & 2.0560906 & Upregulated \\
\hline \multicolumn{4}{|l|}{ Protein binding } \\
\hline NM_021934.3 & C12orf44 & 2.943061 & Upregulated \\
\hline NM_181291.1 & WDR20 & 1.9003692 & Upregulated \\
\hline NM_006207.1 & PDGFRL & 3.129943 & Upregulated \\
\hline \multicolumn{4}{|l|}{ Protein folding } \\
\hline NM_021800.2 & DNAJC12 & 4.182389 & Upregulated \\
\hline \multicolumn{4}{|c|}{ Endoplasmic reticulum unfolded protein response } \\
\hline NM_001010990.1 & HERPUD1 & 6.5408597 & Upregulated \\
\hline \multicolumn{4}{|l|}{ Regulation of RhoGTPase activity } \\
\hline NM_153213.2 & ARHGEF19 & 2.899712 & Upregulated \\
\hline \multicolumn{4}{|l|}{ Regulation of rab GTPase activity } \\
\hline NM_022771.3 & TBC1D15 & 2.361947 & Upregulated \\
\hline NM_020705.1 & $\mathrm{TBC} 1 \mathrm{D} 24$ & 3.0267975 & Upregulated \\
\hline \multicolumn{4}{|l|}{ Cell-cell signalling } \\
\hline NM_001005914.1 & SEMA3B & 1.764685 & Upregulated \\
\hline NM_032331.2 & MGC2408 & 2.6944642 & Upregulated \\
\hline NM_001407.1 & CELSR3 & 3.9751458 & Upregulated \\
\hline \multicolumn{4}{|l|}{ Translation } \\
\hline NM_181463.1 & MRPL55 & 3.1179419 & Upregulated \\
\hline NM_003136.2 & SRP54 & 2.6215558 & Upregulated \\
\hline \multicolumn{4}{|l|}{ Electron transport chain } \\
\hline NM_025147.2 & COQ10B & 1.7724766 & Upregulated \\
\hline NM_007022.3 & CYB561D2 & 3.542795 & Upregulated \\
\hline \multicolumn{4}{|l|}{ Signal transduction } \\
\hline NM_133173.2 & APBB3 & 2.0743015 & Upregulated \\
\hline NM_016115.3 & ASB3 & 2.5060174 & Upregulated \\
\hline \multicolumn{4}{|l|}{ Cell proliferation } \\
\hline NM_206825.1 & GNL3 & 4.2791877 & Upregulated \\
\hline \multicolumn{4}{|l|}{ DNA replication } \\
\hline NM_017443.3 & POLE3 & 3.112311 & Upregulated \\
\hline \multicolumn{4}{|l|}{ Oxidoreductase } \\
\hline NM_017758.2 & ALKBH5 & 3.526197 & Upregulated \\
\hline \multicolumn{4}{|l|}{ Microtubule sitoplamic organization } \\
\hline NM_014171.3 & CRIPT & 2.496879 & Upregulated \\
\hline \multicolumn{4}{|l|}{ Growth } \\
\hline NM_031479.3 & INHBE & 24.436758 & Upregulated \\
\hline \multicolumn{4}{|l|}{ Biosynthesis process } \\
\hline NM_014305.1 & TGDS & 2.4665043 & Upregulated \\
\hline NM_133443.1 & GPT2 & 6.0515475 & Upregulated \\
\hline NM_005768.5 & OACT5 & 2.454743 & Downregulated \\
\hline
\end{tabular}


TABLE 2: Continued.

\begin{tabular}{lccc}
\hline Gene Ontology/accession number & Gene symbol & Fold change & \\
\hline Modification-dependent protein catabolic process & & & Regulation \\
NM_203301.1 & FBXO33 & 1.5563589 & Upregulated \\
NM_015984.1 & UCHL5 & 2.2333875 & Upregulated \\
Hydrolase activity & & & Downregulated \\
$\quad$ NM_203453.2 & PPAPDC2 & 1.8218696 & \\
Proteolysis & & & Downregulated \\
NM_032549.1 & IMMP2L & 1.596754 & \\
\hline
\end{tabular}

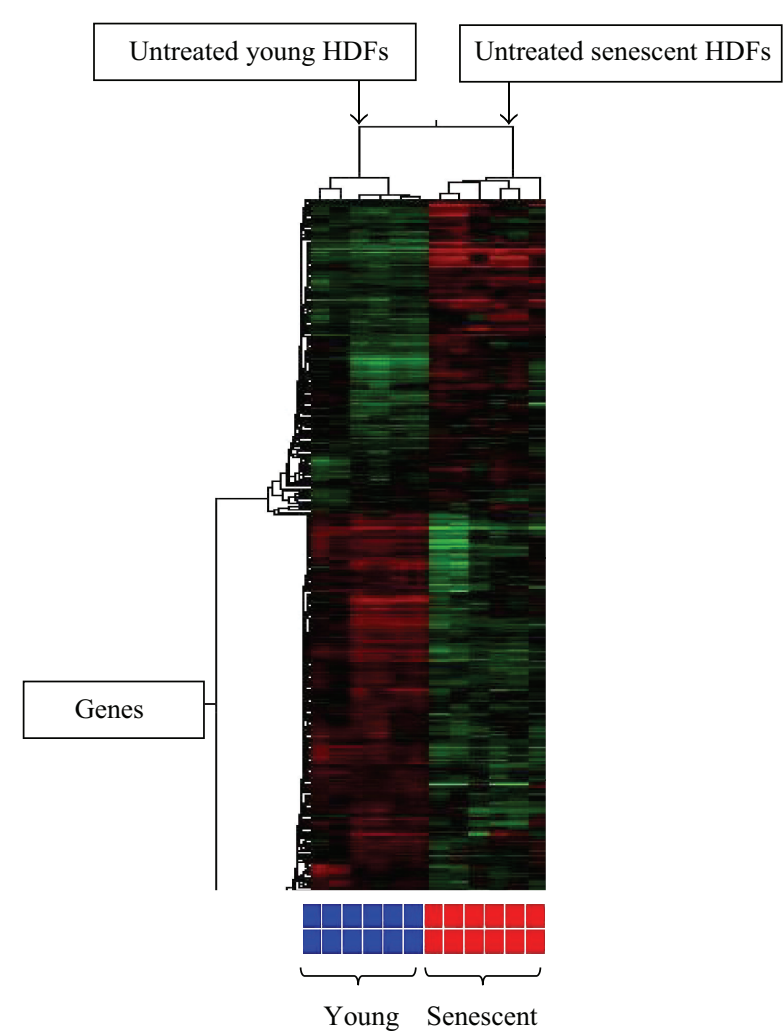

(a)

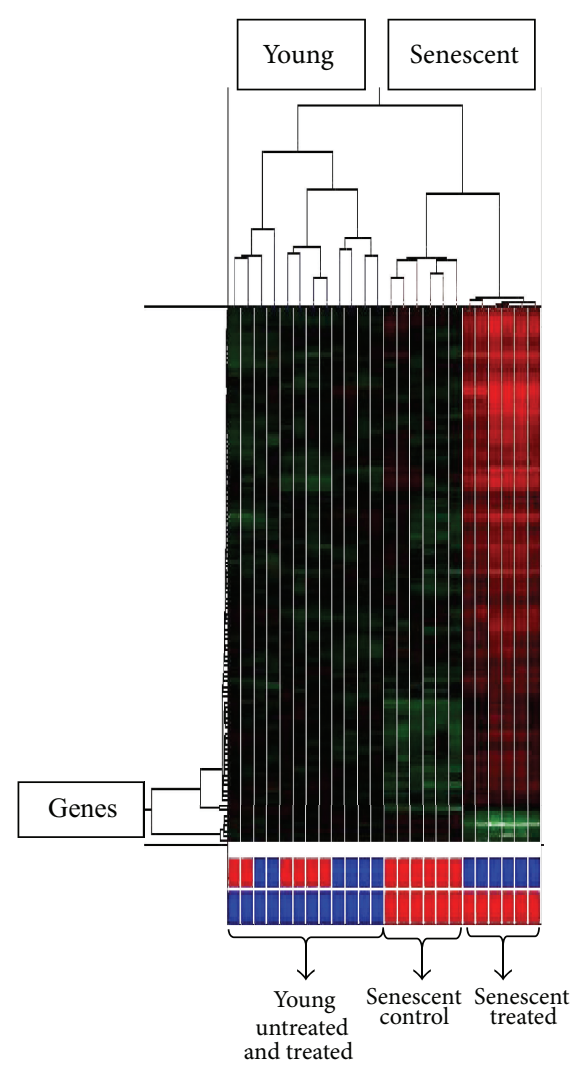

(b)

FIGURE 2: Hierarchical cluster analysis of genes showed the differential expression of genes in young and senescent HDFs (a) and senescent HDFs in response to $\gamma$-tocotrienol treatment (b) $(P<0.001)$. Samples were clustered under their conditions.

senescent HDFs as compared to untreated control senescent HDFs (Figure 4). Comparison between microarray and RTPCR data showed that there was a consistent expression pattern of ARF4, HSPA5, and HERPUD1 genes which are involved in protein transport and negative regulation of apoptosis (Table 4 ).

\section{Discussion}

Nonantioxidant activities of vitamin E particularly $\alpha$ tocopherol has been increasingly reported. For instance, $\alpha$ tocopherol and $\alpha$-tocopheryl phosphate were reported to be involved as mediators of lipid metabolism by modulating signal transduction and gene expression [8]. Our findings from the present study showed that another form of vitamin E, $\gamma$-tocotrienol, was able to modulate gene expression in human diploid fibroblasts. In response to $\gamma$-tocotrienol treatment, a total of 100 genes were differentially expressed in senescent HDFs which included IRAK3, SelS, HSPA5, HERPUD1, DNAJB9, SEPR1, C18orf55, ARF4, RINT1, NXT1, CADPS2, COG6, and GLRX5. Gene Set Enrichment Analysis (GSEA) revealed that IRAK3 was involved in inflammation process specifically in the negative regulation of tumor necrosis factor production and negative regulation of interleukin6 production. IRAK3 encodes for one of the interleukin 


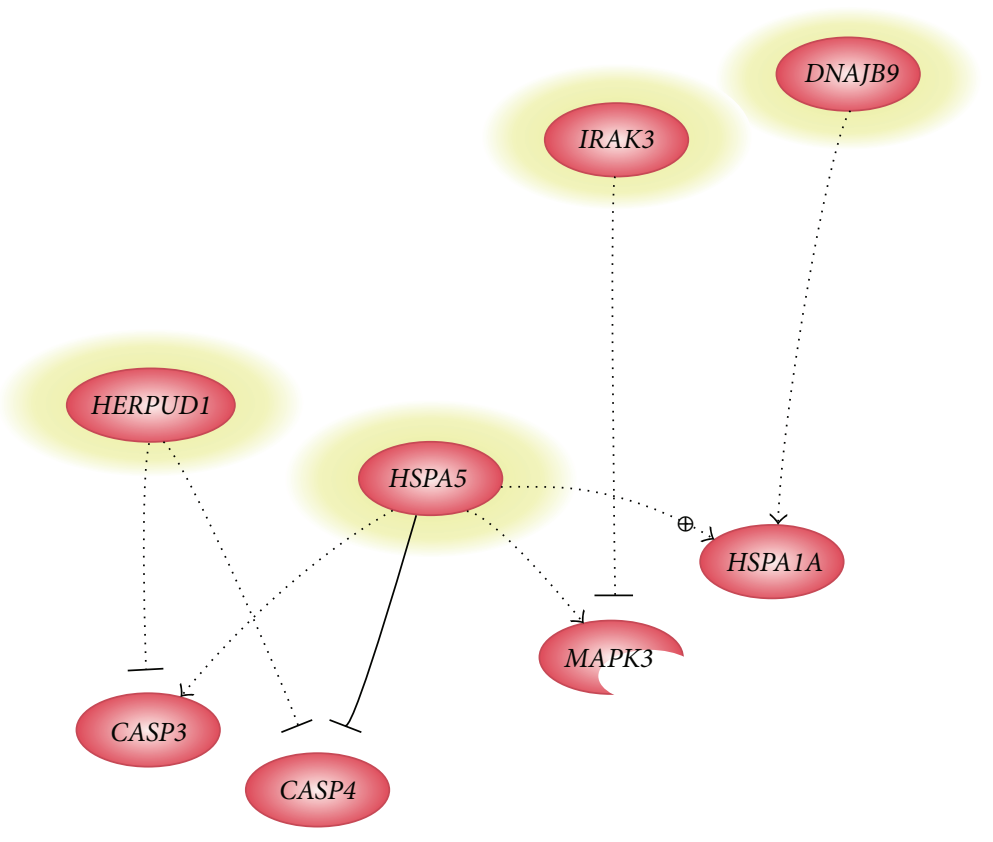

(a)

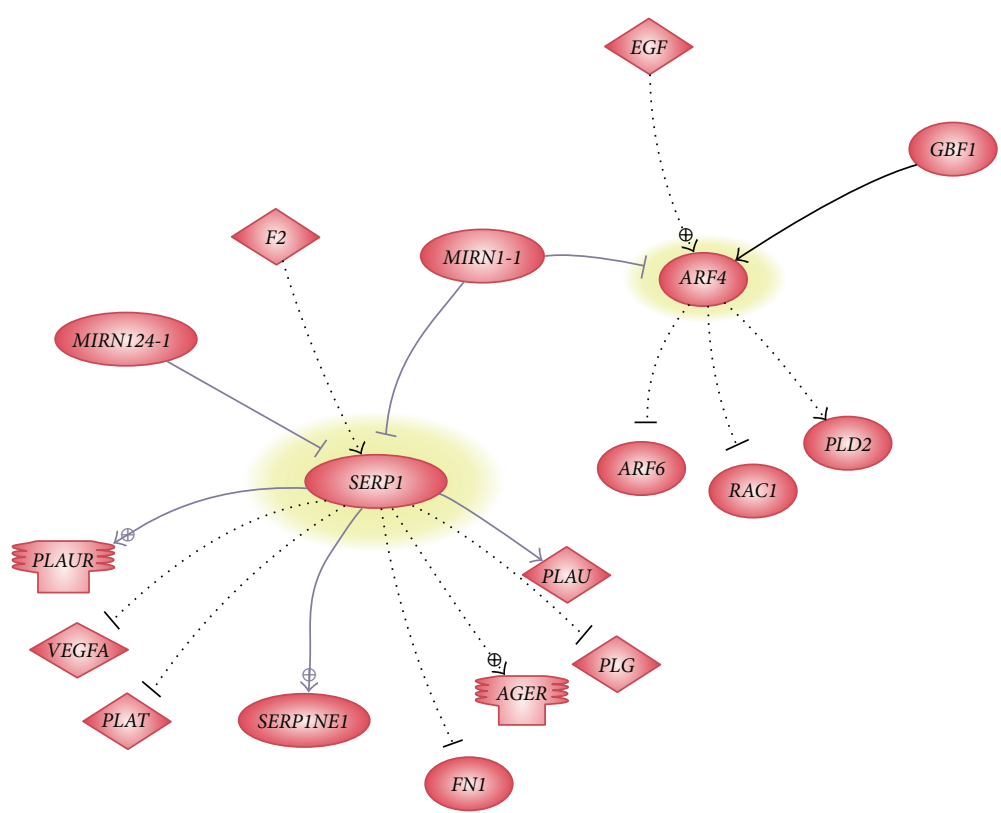

(b)

FIGURE 3: Network of common target for the molecular relationship of selected differentially expressed genes in $\gamma$-tocotrienol-treated senescent HDFs. The highlighted genes were the regulator for the downstream genes.

receptor-associated kinase (IRAK) family and has a role in both positive and negative regulation of signal transduction. This gene is also known as IRAK-M, and previously, the expression of human IRAK-M was limited to monocytes and macrophages [15], However in the present study, its expression was significantly increased in $\gamma$-tocotrienoltreated senescent fibroblast cells.

The aging process is attributed to the presence of low chronic inflammation resulting in a stressed condition. Genes related to inflammation are relevant after taking into account that the innate immunity is more involved during inflammation. A chronic inflammatory status called inflame aging appears to be the major component of common age-related diseases including cardiovascular diseases and infections [16]. Among the inflammatory agents that have been identified were interleukin- 6 , interleukin- $1 \beta$, cyclooxygenase 2 and tumor necrosis factor (TNF). Up-regulation of proinflammatory mediators was observed during aging due to an age-related redox imbalance that activates several proinflammatory signaling pathways. Dysregulation of these 


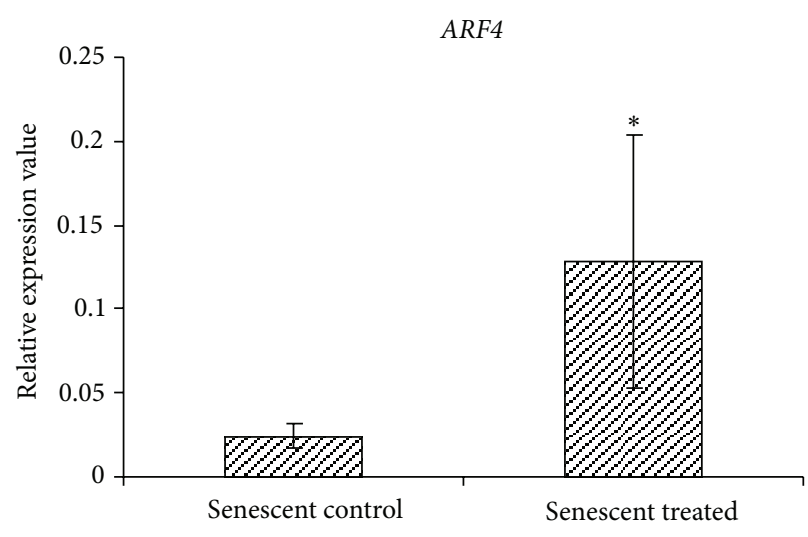

$\square$ ARF4

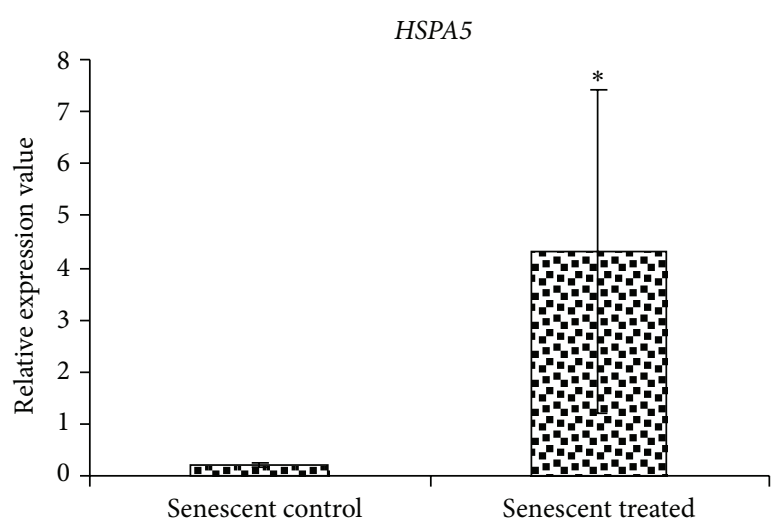

国 HSPA5

(b)

(a)

HERPUD1

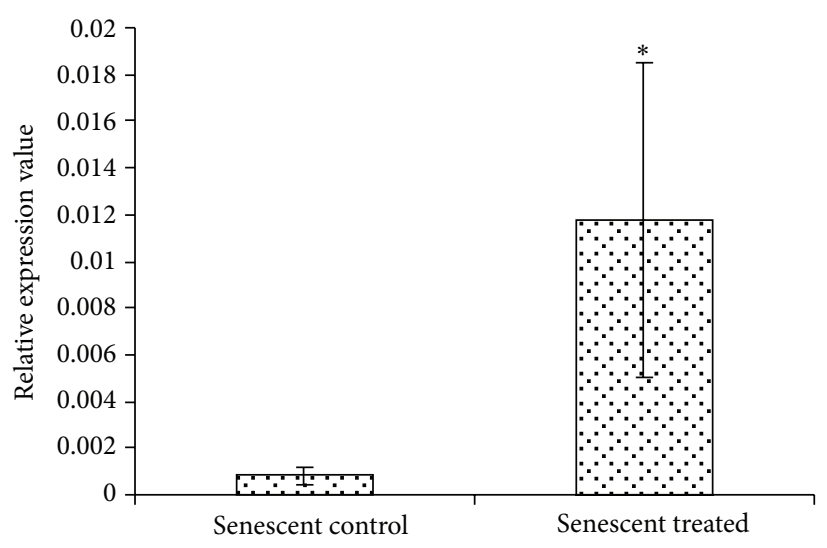

] HERPUD1

(c)

FIGURE 4: Based on pathway analysis, three genes were selected for validation by qRT-PCR technique. All data were normalized to the housekeeping gene, GAPDH. The expression patterns obtained through qRT-PCR were consistent with the microarray results. ARF4 (a), HSPA5 (b), and HERPUD1 (c) were significantly upregulated in $\gamma$-tocotrienol-treated senescent HDFs compared to untreated control senescent HDFs $(P<0.05)$.

cytokines promotes inflammation and tissue damage. This indicated that aging is accompanied by chronic low-grade inflammation state showed by 2 -to 4 -fold increase in serum levels of inflammatory mediators such as C-reactive protein, tumor necrosis factor (TNF) and cyclooxygenase 2 (COX2) [17]. Up-regulation of IRAK3 in this study may indicate protection against cellular aging and age-related diseases.

In the present study we also found that selenoprotein $S$ (SelS) was significantly upregulated in $\gamma$-tocotrienol-treated senescent fibroblast cells. Selenoprotein $S$ is involved in negative regulation of tumor necrosis factor production, negative regulation of interleukin- 6 production, and cell redox homeostasis. It encodes for an endoplasmic reticulum (ER) transmembrane protein and is present in a variety of tissues such as liver, skeletal muscle, and adipose tissue [18]. Selenoproteins contain the twenty-first amino acid, selenocysteine, reported to be involved in cellular defenses against oxidative damage. Furthermore, these proteins are involved in important metabolic and developmental pathways in response to environmental challenges [19]. Many of the selenoproteins are involved in protection against oxidative stress or in maintaining cellular redox balance. SelS is considered as an important component of retrotranslocation channel in endoplasmic reticulum-associated protein degradation (ERAD). This ER membrane protein functions in stress responses to prevent the deleterious consequences of accumulation of misfolded proteins which has been linked to immune and inflammatory processes. Previous findings have suggested that SelS may regulate cytokine production in macrophages, and a regulatory loop between cytokines and SelS has been proposed to play a key role in controlling the inflammatory response [20]. Negative regulation of TNF and interleukin- 6 production by IRAK3 and SelS in $\gamma$-tocotrienoltreated senescent HDFs observed in this study may indicate inhibition of chronic inflammatory processes that normally accompanies cellular aging. Thus this may suggest one of 
TABLE 3: Selected significant biological processes that were modulated in senescent HDFs after $\boldsymbol{\gamma}$-tocotrienol treatment for $24 \mathrm{~h}$.

\begin{tabular}{|c|c|c|c|}
\hline \multirow{2}{*}{ Biological process } & \multicolumn{3}{|c|}{ Gene Set Enrichment Analysis (GSEA) } \\
\hline & $\begin{array}{l}\text { Normalized } \\
\text { Enrichment } \\
\text { Score }\end{array}$ & Gene symbol & Description \\
\hline \multirow[t]{3}{*}{$\begin{array}{l}\text { Negative regulation of tumor necrosis factor } \\
\text { production }\end{array}$} & 1.54164 & & \\
\hline & & IRAK3 & Interleukin-1 receptor-associated kinase 3 \\
\hline & & Sels & selenoprotein S \\
\hline \multirow[t]{3}{*}{$\begin{array}{l}\text { Negative regulation of interleukin-6 } \\
\text { production }\end{array}$} & 1.65684 & & \\
\hline & & IRAK3 & Interleukin-1 receptor-associated kinase 3 \\
\hline & & Sels & selenoprotein S \\
\hline \multirow[t]{3}{*}{ Negative regulation of caspase activity } & 1.40798 & & \\
\hline & & HSPA5 & $\begin{array}{l}\text { Heat shock } 70 \mathrm{kDa} \text { protein } 5 \\
\text { (glucose-regulated protein, } 78 \mathrm{kDa} \text { ) }\end{array}$ \\
\hline & & HERPUD1 & $\begin{array}{l}\text { Homocysteine-inducible, endoplasmic } \\
\text { reticulum stress-inducible, ubiquitin-like } \\
\text { domain member } 1\end{array}$ \\
\hline \multirow[t]{5}{*}{ Response to stress } & 1.61171 & & \\
\hline & & HSPA5 & $\begin{array}{l}\text { Heat shock } 70 \mathrm{kDa} \text { protein } 5 \\
\text { (glucose-regulated protein, } 78 \mathrm{kDa} \text { ) }\end{array}$ \\
\hline & & HERPUD1 & $\begin{array}{l}\text { Homocysteine-inducible, endoplasmic } \\
\text { reticulum stress-inducible, ubiquitin-like } \\
\text { domain member } 1\end{array}$ \\
\hline & & DNAJB9 & $\begin{array}{l}\text { DnaJ (Hsp40) homolog, subfamily B, } \\
\text { member } 9\end{array}$ \\
\hline & & SERP1 & $\begin{array}{l}\text { Stress-associated endoplasmic reticulum } \\
\text { protein } 1\end{array}$ \\
\hline \multirow[t]{8}{*}{ Protein transport } & 1.68316 & & \\
\hline & & C18orf55 & Chromosome 18 open reading frame 55 \\
\hline & & ARF4 & ADP-ribosylation factor 4 \\
\hline & & RINT1 & RAD50 interactor 1 \\
\hline & & SERP1 & $\begin{array}{l}\text { Stress-associated endoplasmic reticulum } \\
\text { protein } 1\end{array}$ \\
\hline & & NXT1 & NTF2-like export factor 1 \\
\hline & & CADPS2 & $\begin{array}{l}\mathrm{Ca}^{2+} \text {-dependent activator protein for } \\
\text { secretion } 2\end{array}$ \\
\hline & & COG6 & Component of oligomeric golgi complex 6 \\
\hline \multirow[t]{3}{*}{ Cell redox homeostasis } & 1.56752 & & \\
\hline & & GLRX5 & Glutaredoxin 5 homolog \\
\hline & & Sels & Selenoprotein S \\
\hline
\end{tabular}

the mechanisms involved for $\gamma$-tocotrienol in slowing down cellular aging of HDFs.

$\gamma$-Tocotrienol also modulated the expression of genes that are involved in protein transport. Our results showed that ADP-ribosylation factor $4(A R F 4)$ was significantly upregulated in $\gamma$-tocotrienol-treated senescent HDFs. ADPribosylation factors have been implicated in several important cellular processes, including membrane trafficking and activation of phospholipase D [21]. They are members of the Ras super family of small guanine nucleotide-binding proteins and were initially identified as proteins that stimulate the ADP-ribosyl transferase activity of cholera toxin in vitro. ARFs are ubiquitously expressed from yeast to mammals and function primarily in the regulation of membrane trafficking. Based on their deduced amino acid sequence, protein size, and phylogenetic analysis, these proteins can be divided into three groups which are class I (ARF1, ARF2, and ARF3), class II (ARF4, ARF5), and class III (ARF6). Previous report 
TABLE 4: Comparison between microarray and qRT-PCR data.

\begin{tabular}{lccc}
\hline Gene & $\begin{array}{c}\text { Fold change } \\
\text { (RT PCR) }\end{array}$ & $\begin{array}{c}\text { Fold change } \\
\text { (microarray) }\end{array}$ & Regulation \\
\hline ARF4 & 5.275 & 3.66465 & Upregulated \\
HERPUD1 & 14.26 & 6.54086 & Upregulated \\
HSPA5 & 20.99 & 5.95136 & Upregulated \\
\hline
\end{tabular}

has demonstrated that overexpression of ARF4 in U373MG cells suppresses $\mathrm{N}$-(4-hydroxyphenyl) retinamide (4-HPR)induced cell death. The findings from yeast-based functional screening in $S$. cerevisiae which facilitates the identification of antiapoptotic mammalian genes showed that ARF4 may function as a novel suppressor of apoptosis [22].

Heat shock proteins (Hsps) have been studied for many years, and there are evidences that demonstrated the role of Hsp up-regulation in tissues and cell protection in a wide variety of stress conditions. Heat shock proteins (HSPs) belong to a class of highly conserved proteins that act physiologically as molecular chaperones to stabilize existing proteins against aggregation and mediate the folding of newly translated proteins in the cytosol and other organelles [23]. Additionally, they have been shown to demonstrate antiapoptotic effects against a wide range of both physical and chemical apoptotic stimuli [24]. Similar finding was found in our study, whereby the HSPA5 (heat shock $70 \mathrm{kDa}$ protein 5) also known as BiP or GRP78 (glucose-regulated protein, $78 \mathrm{kDa}$ ) which is involved in negative regulation of caspase activity in response to stress was significantly increased in $\gamma$ tocotrienol-treated senescent HDFs. Besides, treatment with $\gamma$-tocotrienol in senescent HDFs caused up-regulation of HERPUD1 which is also involved in negative regulation of caspase activity in response to stress. HERP (homocysteineinduced ER protein) which was recently identified and characterized as an ER membrane protein was upregulated in response to ER stress. Induction of HERP was reported to be involved in the protection of cells against ER stress. HERP stabilizes ER $\mathrm{Ca}^{2+}$ homeostasis and mitochondrial functions in neuronal cells during ER stress [25]. Thus the findings from the present study showed that treatment with $\gamma$-tocotrienol in senescent HDFs may delay cellular aging of HDFs by modulating cellular stress responses and regulating the apoptosis pathway.

Oxidative stress is known to be involved in a number of pathological conditions, including neurodegeneration, cardiovascular disease, and stroke, and even plays a role in natural aging. Oxidative stress occurs when the levels of oxidants are higher than the levels of antioxidants, thus overwhelming the system. The imbalance between prooxidants and antioxidants leads to an accumulation of oxidative damage with age in a variety of macromolecules resulting in a progressive loss of functional cellular processes, leading to the aging phenotype [26]. A progressive rise of oxidative stress due to the altered redox homeostasis appears to be one of the hallmarks of the aging process. Changes in the pattern of gene expression through ROS-sensitive transcription factors give rise to both aging and inflammation phenotypes. Chronic oxidative stress and inflammatory reactions lead to many ageassociated diseases such as atherosclerosis and arthritis [27].

Cells have developed both nonenzymatic and enzymatic defense mechanisms to counteract the deleterious effects of oxidative stress by either detoxifying reactive oxygen species (ROS) or repairing ROS-induced damage. Nonenzymatic examples of antioxidant include vitamin C, vitamin E, ubiquinone, flavonoids and glutathione (GSH), and examples of enzymatic scavengers include catalase, glutathione peroxidase, thioredoxin, $\mathrm{Cu} / \mathrm{Zn}$ superoxide dismutase $(\mathrm{Cu} / \mathrm{Zn}$ $\mathrm{SOD}), \mathrm{Mn}$ /superoxide dismutase (MnSOD), and glutaredoxins [28]. Interestingly, our finding showed that $\gamma$-tocotrienol intervention in senescent HDFs caused increased expression of glutaredoxin 5 homolog (GLRX5) gene which is involved in cell redox homeostasis. Glutaredoxins are glutathionedependent oxidoreductases that help in maintaining cellular redox homeostasis in the cell. The glutaredoxin system consists of GSH, NADPH, and GSH reductase. The mammalian system contains three known members of the glutaredoxin family Grx1, Grx2, and Grx5. The recently discovered glutaredoxin 5 , a monothiol glutaredoxin, is hypothetically localized to the mitochondria. The loss of Grx5 in yeast leads to constitutive oxidative damage, sensitization to ROS, iron accumulation and inactivation of iron-sulfur (Fe-S) cluster containing enzymes [29]. Our findings are in agreement with a recent study that showed overexpression of glutaredoxin 5 caused decreased in DNA fragmentation and protect cells against $\mathrm{H}_{2} \mathrm{O}_{2}$ induced apoptosis and ROS formation [30].

In summary, the findings from this study elucidated the nonantioxidant properties of a vitamin $\mathrm{E}$ isomer, $\gamma$ tocotrienol in delaying cellular aging indicated by regulation of protective biological processes through the modulation of gene expression in human diploid fibroblasts.

\section{Conclusion}

$\gamma$-Tocotrienol may delay or protect against cellular aging of human diploid fibroblasts by modulating genes expression that are involved in biological processes related to oxidative stress such as inflammation, protein transport, apoptosis, and cell redox homeostasis.

\section{Conflict of Interests}

The authors declare that they have no conflict of interests.

\section{Acknowledgments}

This study was funded by the Ministry of Higher Learning under the Fundamental Research Grant Scheme UKMFF-03-FRGS0043-2009 and Universiti Kebangsaan Malaysia Grant FF-328-2009.

\section{References}

[1] I. P. Trougakos, A. Saridaki, G. Panayotou, and E. S. Gonos, "Identification of differentially expressed proteins in senescent human embryonic fibroblasts," Mechanisms of Ageing and Development, vol. 127, no. 1, pp. 88-92, 2006. 
[2] T. B. Kirkwood, "Evolution of ageing," Mechanisms of Ageing and Development, vol. 123, no. 7, pp. 737-745, 2002.

[3] I. K. Yoon, H. K. Kim, Y. K. Kim et al., "Exploration of replicative senescence-associated genes in human dermal fibroblasts by cDNA microarray technology," Experimental Gerontology, vol. 39, no. 9, pp. 1369-1378, 2004.

[4] B. Braam, M. Langelaar-Makkinje, A. Verkleij et al., "Antioxidant sensitivity of donor age-related gene expression in cultured fibroblasts," European Journal of Pharmacology, vol. 542, no. 1-3, pp. 154-161, 2006.

[5] S. Makpol, L. W. Durani, K. H. Chua, Y. A. Mohd Yusof, and W. Z. Wan Ngah, "Tocotrienol-rich fraction prevents cell cycle arrest and elongates telomere length in senescent human diploid fibroblasts," Journal of Biomedicine and Biotechnology, vol. 2011, Article ID 506171, 2011.

[6] H. Zhang, K. H. Pan, and S. N. Cohen, "Senescence-specific gene expression fingerprints reveal cell-type-dependent physical clustering of up-regulated chromosomal loci," Proceedings of the National Academy of Sciences of the United States of America, vol. 100, no. 6, pp. 3251-3256, 2003.

[7] M. L. Failla, "Trace elements and host defense: recent advances and continuing challenges," Journal of Nutrition, vol. 133, no. 5, supplement 1, pp. 1443S-1447S, 2003.

[8] J. M. Zingg, R. Libinaki, C. Q. Lai et al., "Modulation of gene expression by $\alpha$-tocopherol and $\alpha$-tocopheryl phosphate in THP-1 monocytes," Free Radical Biology and Medicine, vol. 49, no. 12, pp. 1989-2000, 2010.

[9] Y. K. Nakamura and S. T. Omaye, "Vitamin E-modulated gene expression associated with ROS generation," Journal of Functional Foods, vol. 1, no. 3, pp. 241-252, 2009.

[10] C. K. Sen, S. Khanna, and S. Roy, "Tocotrienols in health and disease: the other half of the natural vitamin E family," Molecular Aspects of Medicine, vol. 28, no. 5-6, pp. 692-728, 2007.

[11] J. P. Kamat and T. P. A. Devasagayam, "Tocotrienols from palm oil as potent inhibitors of lipid peroxidation and protein oxidation in rat brain mitochondria," Neuroscience Letters, vol. 195, no. 3, pp. 179-182, 1995.

[12] A. A. Qureshi, B. A. Bradlow, W. A. Salser, and L. D. Brace, "Novel tocotrienols of rice bran modulate cardiovascular disease risk parameters of hypercholesterolomic humans," Journal of Nutritional Biochemistry, vol. 8, no. 5, pp. 290-298, 1997.

[13] S. Makpol, A. Z. Abidin, K. Sairin, M. Mazlan, G. M. Top, and W. Z. W. Ngah, " $\gamma$-tocotrienol prevents oxidative stressinduced telomere shortening in human fibroblasts derived from different aged individuals," Oxidative Medicine and Cellular Longevity, vol. 3, no. 1, pp. 35-43, 2010.

[14] S. Makpol, A. Zainuddin, K. H. Chua, Y. A. M. Yusof, and W. Z. W. Ngah, "Gamma-tocotrienol modulation of senescenceassociated gene expression prevents cellular aging in human diploid fibroblasts," Clinics, vol. 67, no. 2, pp. 135-143, 2012.

[15] S. Flannery and A. G. Bowie, "The interleukin-1 receptorassociated kinases: critical regulators of innate immune signalling," Biochemical Pharmacology, vol. 80, no. 12, pp. 19811991, 2010.

[16] C. Franceschi, M. Bonafè, S. Valensin et al., "Inflamm-aging. An evolutionary perspective on immunosenescence," Annals of the New York Academy of Sciences, vol. 908, pp. 244-254, 2000.

[17] H. Y. Chung, M. Cesari, S. Anton et al., "Molecular inflammation: underpinnings of aging and age-related diseases," Ageing Research Reviews, vol. 8, no. 1, pp. 18-30, 2009.
[18] S. Du, H. Liu, and K. Huang, "Influence of SelS gene silence on $\beta$-Mercaptoethanol-mediated endoplasmic reticulum stress and cell apoptosis in HepG2 cells," Biochimica et Biophysica Acta, vol. 1800, no. 5, pp. 511-517, 2010.

[19] Z. R. Stoytcheva and M. J. Berry, "Transcriptional regulation of mammalian selenoprotein expression," Biochimica et Biophysica Acta, vol. 1790, no. 11, pp. 1429-1440, 2009.

[20] Y. Gao, N. R. F. Hannan, S. Wanyonyi et al., "Activation of the selenoprotein SEPS1 gene expression by pro-inflammatory cytokines in HepG2 cells," Cytokine, vol. 33, no. 5, pp. 246-251, 2006.

[21] S. W. Kim, M. Hayashi, J. F. Lo, Y. Yang, J. S. Yoo, and J. D. Lee, "ADP-ribosylation factor 4 small GTPase mediates epidermal growth factor receptor-dependent phospholipase D2 activation," Journal of Biological Chemistry, vol. 278, no. 4, pp. 2661-2668, 2003.

[22] I. S. Woo, S. Y. Eun, H. S. Jang et al., "Identification of ADP-ribosylation factor 4 as a suppressor of N-(4hydroxyphenyl)retinamide-induced cell death," Cancer Letters, vol. 276, no. 1, pp. 53-60, 2009.

[23] Y. Y. Lee, K. T. K. Wong, J. Tan et al., "Overexpression of heat shock proteins (HSPs) in CHO cells for extended culture viability and improved recombinant protein production," Journal of Biotechnology, vol. 143, no. 1, pp. 34-43, 2009.

[24] C. Garrido, S. Gurbuxani, L. Ravagnan, and G. Kroemer, "Heat shock proteins: endogenous modulators of apoptotic cell death," Biochemical and Biophysical Research Communications, vol. 286, no. 3, pp. 433-442, 2001.

[25] S. L. Chan, W. Fu, P. Zhang et al., "Herp stabilizes neuronal Ca2+ homeostasis and mitochondrial function during endoplasmic reticulum stress," Journal of Biological Chemistry, vol. 279, no. 27, pp. 28733-28743, 2004.

[26] M. Ott, V. Gogvadze, S. Orrenius, and B. Zhivotovsky, "Mitochondria, oxidative stress and cell death," Apoptosis, vol. 12, no. 5, pp. 913-922, 2007.

[27] Y. Lavrovsky, B. Chatterjee, R. A. Clark, and A. K. Roy, "Role of redox-regulated transcription factors in inflammation, aging and age-related diseases," Experimental Gerontology, vol. 35, no. 5, pp. 521-532, 2000.

[28] J. L. Martindale and N. J. Holbrook, "Cellular response to oxidative stress: signaling for suicide and survival," Journal of Cellular Physiology, vol. 192, no. 1, pp. 1-15, 2002.

[29] M. T. Rodríguez-Manzaneque, J. Tamarit, G. Bellí, J. Ros, and E. Herrero, "Grx5 is a mitochondrial glutaredoxin required for the activity of iron/sulfur enzymes," Molecular Biology of the Cell, vol. 13, no. 4, pp. 1109-1121, 2002.

[30] G. R. Linares, W. Xing, K. E. Govoni, S. T. Chen, and S. Mohan, "Glutaredoxin 5 regulates osteoblast apoptosis by protecting against oxidative stress," Bone, vol. 44, no. 5, pp. 795-804, 2009. 


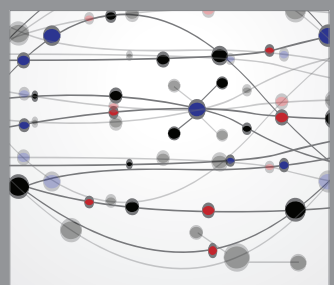

The Scientific World Journal
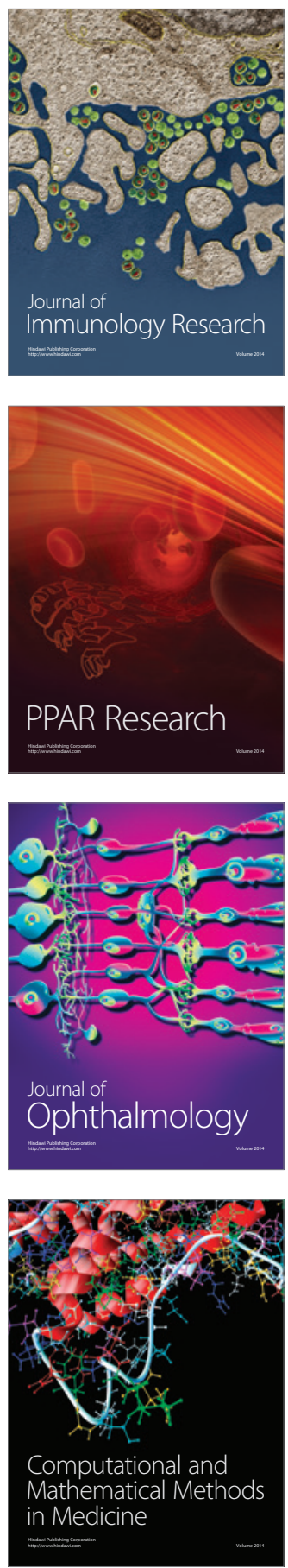

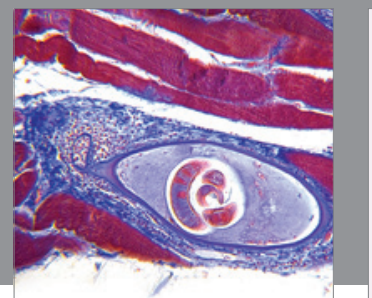

Gastroenterology

Research and Practice
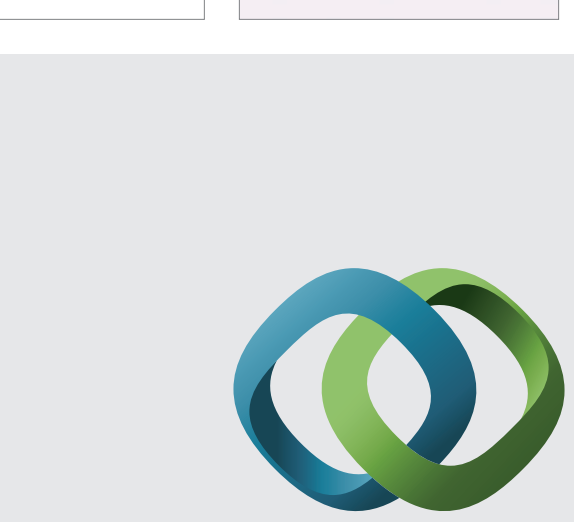

\section{Hindawi}

Submit your manuscripts at

http://www.hindawi.com
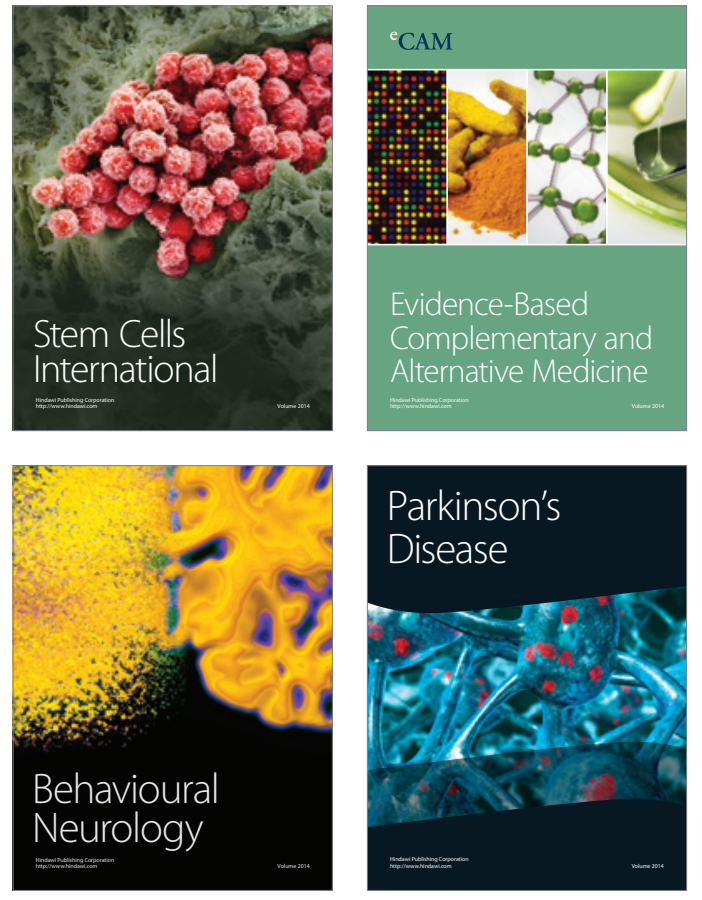
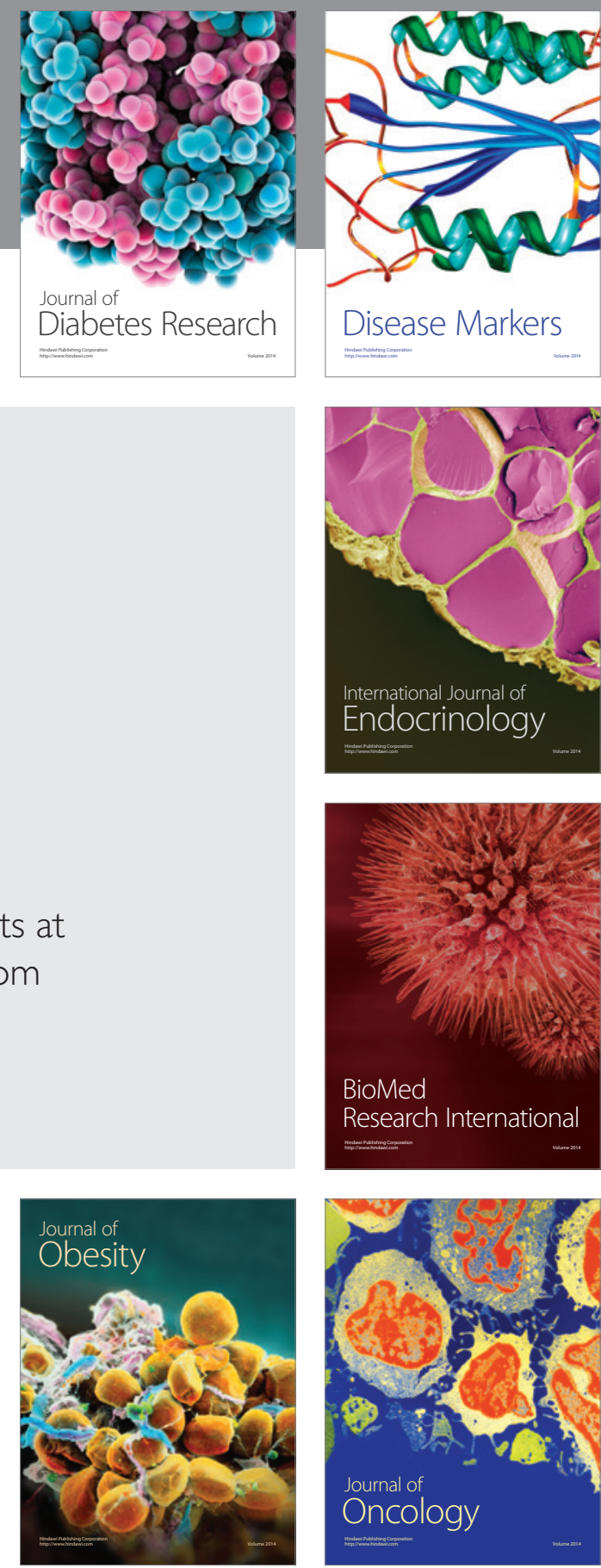

Disease Markers
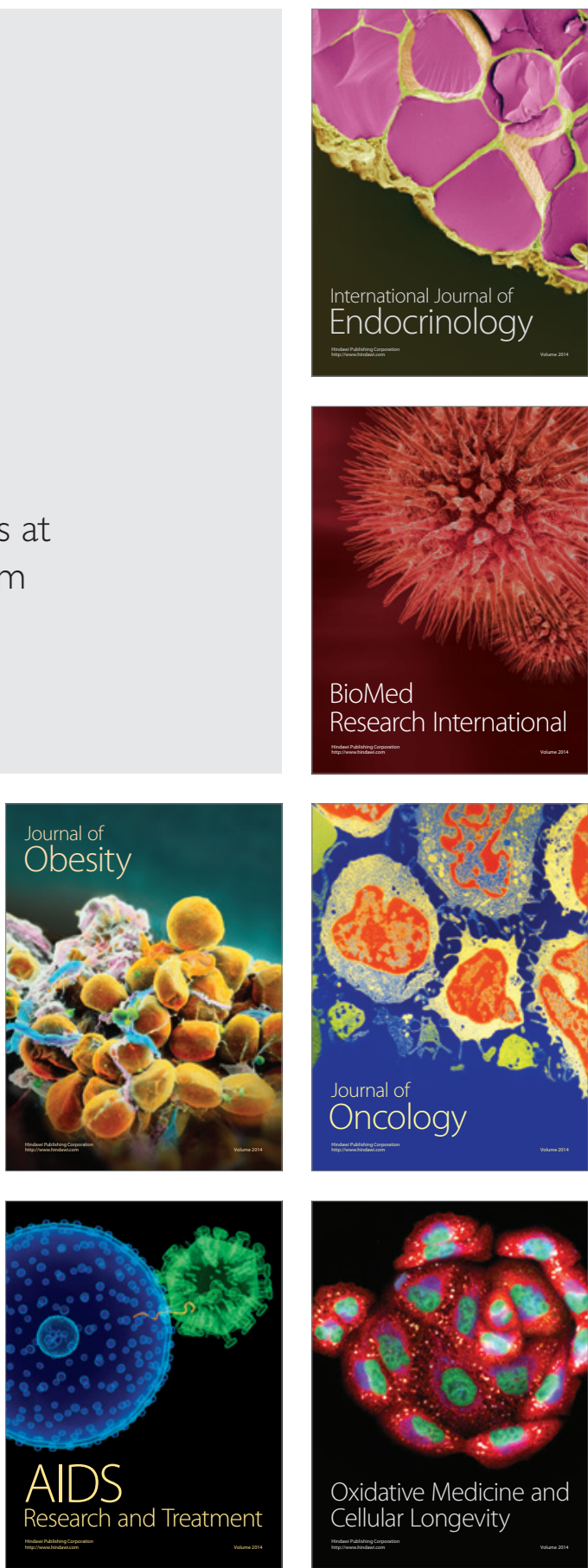